\title{
Simultaneous Tuberculous Infection of Lung and Allograft in Renal Transplant
}

\author{
Hwang $\mathrm{E}^{{ }_{1}}$ and Hwang $\mathrm{I}^{2}$
}

${ }^{1}$ Department of Internal Medicine, Kidney Institute, Keimyung University School of Medicine, Daegu, Republic of Korea ${ }^{2}$ Department of Pathology, Keimyung University School of Medicine, Daegu, Republic of Korea

*Corresponding author: Hwang E, M.D., Ph.D., Kidney Institute, Keimyung University 1095 Dalgubeol-daero, DalseoGu, Daegu 42601, Republic of Korea, Fax: +82-53-255-9703, Tel: +82-53-258-7396, E-mail: eahwang@gmail.com

Citation: Hwang E, Hwang I (2019) Simultaneous Tuberculous Infection of Lung and Allograft in Renal Transplant. J Nephrol Kidney Dis 1(1): 104. doi: 10.15744/2767-9225.1.104

Received Date: November 15, 2019 Accepted Date: April 28, 2020 Published Date: April 30, 2020

\begin{abstract}
Tuberculosis is common infectious complication in kidney transplant recipients. In immunosuppressed patients, clinical manifestations of tuberculosis are varied and delayed diagnosis and poor clinical outcomes. Especially allograft involvement of tuberculosis can cause allograft loss. In this report, we present the case of 46-year-old man diagnosed disseminated tuberculosis involving allograft kidney successfully treated with maintenance of allograft function.
\end{abstract}

Keywords: Allograft; Tuberculosis; Transplant

\section{Introduction}

Tuberculosis (TB) is known as a common opportunistic infection in immunosuppressed patients such as organ transplantation recipients. Incidence of TB in transplant recipients is high compared to the general population by 20-74 times and fatality of $\mathrm{TB}$ in these patients is not rare [1,2]. Though, lungs are the most common site for TB in kidney transplant (KT) recipients, extrapulmonary involvement is common. Extrapulmonary disease can involve gastrointestinal, bone marrow, lymph nodes, skin, allograft kidney or disseminated form [2]. The clinical presentations of TB in transplant recipients are atypical, often leading to a delayed diagnosis and poor outcomes. Especially delayed diagnosis of disseminated TB involving allograft kidney can cause allograft loss or patient death. We report a case of lung and allograft kidney TB, successfully treated without graft loss.

\section{Case report}

A 46-year-old man with end-stage renal disease due to Immunoglobulin A (IgA) nephropathy received first KT as renal replacement therapy 17 years ago. The patient diagnosed of IgA nephropathy by renal biopsy and received KT from mother. Two years after first KT, pulmonary tuberculosis was developed and he received 6-month anti-TB therapy on a 4-drug regimen: isoniazid (INH), rifampin (RFP), pyrazinamide (PZA), and ethambutol (EMB). Five years after KT, the patient returned to hemodialysis therapy after loss of kidney allograft function due to chronic rejection. Ten years ago, he was received second KT from deceased donor. The patient received tacrolimus (Tac), mycophenolate mofetil (MMF) and prednisolone (PN) as immunosuppressive treatment. He received fluconazole, bactrim as posttransplant prophylaxis and also received INH prophylaxis for 12 months for TB prophylaxis. Three weeks after KT, he discharged from the hospital with serum creatinine $(\mathrm{Cr})$ of $1.2 \mathrm{mg} / \mathrm{dL}$. The maintenance immunosuppression consisted of tacrolimus (Tac) with target trough level $8-10 \mathrm{ng} / \mathrm{mL}$ and mycophenolate mofetil (MMF) $750 \mathrm{mg}$ twice daily and prednisolone (PN) $10 \mathrm{mg}$ once a day. Four months after KT, his serum Cr level gradually increased to $2.3 \mathrm{mg} / \mathrm{dL}$ and allograft biopsy was performed. Histological findings revealed chronic rejection and as a result, the intensity of immunosuppression was not reduced. Thereafter, serum Cr level was maintained in the range of 1.8-2.2 mg/dL. Eight month ago, he complains of febrile sensation with coughing. At the time of admission, immunosuppressive therapy consisted of Tac 1.5 mg twice a day (trough level; $6.9 \mathrm{ng} / \mathrm{mL}$ ), MMF $750 \mathrm{mg}$ twice a day and PN $5 \mathrm{mg}$ daily. He diagnosed of cytomegalovirus (CMV) pneumonia by computed tomography (CT) and bronchoscopic biopsy. Intravenous ganciclovir treatment was started with discontinuation of MMF. Follow up CMV PCR with bronchoscopic aspiration lavage was negative after one month treatment of ganciclovir. Seven months after completing ganciclovir therapy, he was admitted with febrile sensation and coughing for 2 weeks. He was received $2 \mathrm{mg}$ of Tac and $5 \mathrm{mg}$ of PN. On physical examination, body temperature (BT) was $38.4{ }^{\circ} \mathrm{C}$. Breathing sound was relatively clear and there was no tenderness on allograft kidney. Laboratory tests showed blood white blood cell (WBC) 8250/ $\mathrm{LL}$ (neutrophils $91.2 \%$ ), hemoglobin 


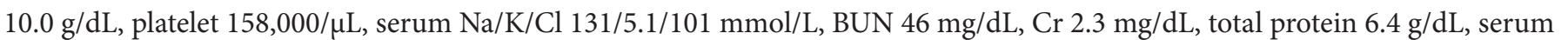
albumin $3.5 \mathrm{~g} / \mathrm{dL}$, total cholesterol $145 \mathrm{mg} / \mathrm{dL}$, C-reactive protein (CRP) $13.5 \mathrm{mg} / \mathrm{dL}$, trough level of Tac $4.7 \mathrm{ng} / \mathrm{ml}$, respectively. In urinary analysis, urine albumin was $25 \mathrm{mg}$ with 2-4 RBCs/HPF, 0-1 WBCs/HPF. In chest radiograph, multiple fine granules were observed in both lung fields (Figure 1). Three morning sputum specimens showed acid-fast bacilli (AFB), however urinary specimens did not showed AFB. Susceptibility testing of the isolate showed all sensitive to INH, RFP, PZA and EMB. He received anti-TB treatment with 4-drugs regimen with discontinuation of $\mathrm{PN}$. On admission day of 10 th, BT was statained over $38.0^{\circ} \mathrm{C}$ and qualitative CMV-PCR test with blood revealed positive for CMV. Intravenous ganciclovir treatment was added. Three weeks after anti-TB medication, sputum AFB smears were negative on three consecutive days. However, despite of anti-TB treatment with ganciclovir treatment for a month, fever was sustained with elevated CRP level of $20.1 \mathrm{mg} / \mathrm{dL}$ and serum Cr increased to 3.9 $\mathrm{mg} / \mathrm{dL}$. On admission day of 30th, an ultrasonography guided graft biopsy was performed. Several vague granulomas (arrows) were present on graft biopsy on microscopic examination (Figure 2a). Granuloma showed a central necrosis and epithelioid cells (arrows) with some lymphocytes few neutrophils (Figure 2b). Serum Cr was increased to $6.0 \mathrm{mg} / \mathrm{dL}$ on day of $32 \mathrm{nd}$, and then slowly improved (Figure 3). The patient became afebrile on 38th days and discharged from the hospital on fifth hospital days. The patient was received Tac $1 \mathrm{mg}$ twice a day as immunosuppresive agent at the time of discharge and serum Cr was stablized to 2.0-2.2 mg/dL. After 1-year anti-TB treatment, multiple fine granules were resolved on follow up chest CT (Figure 4) and serum Cr level was $3.0 \mathrm{mg} / \mathrm{dL}$. Thereafter, allograft function was declined gradually and 5 years after TB infection, the patient started to hemodialysis (HD), three times a week. Currently he is undergoing HD treatment without infectious complications.

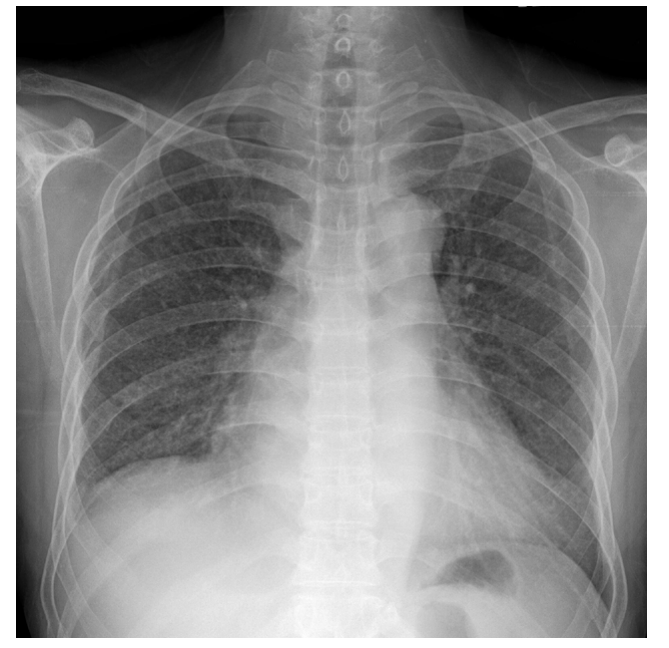

Figure 1: X-ray finding. Multiple tiny nodules in both lungs

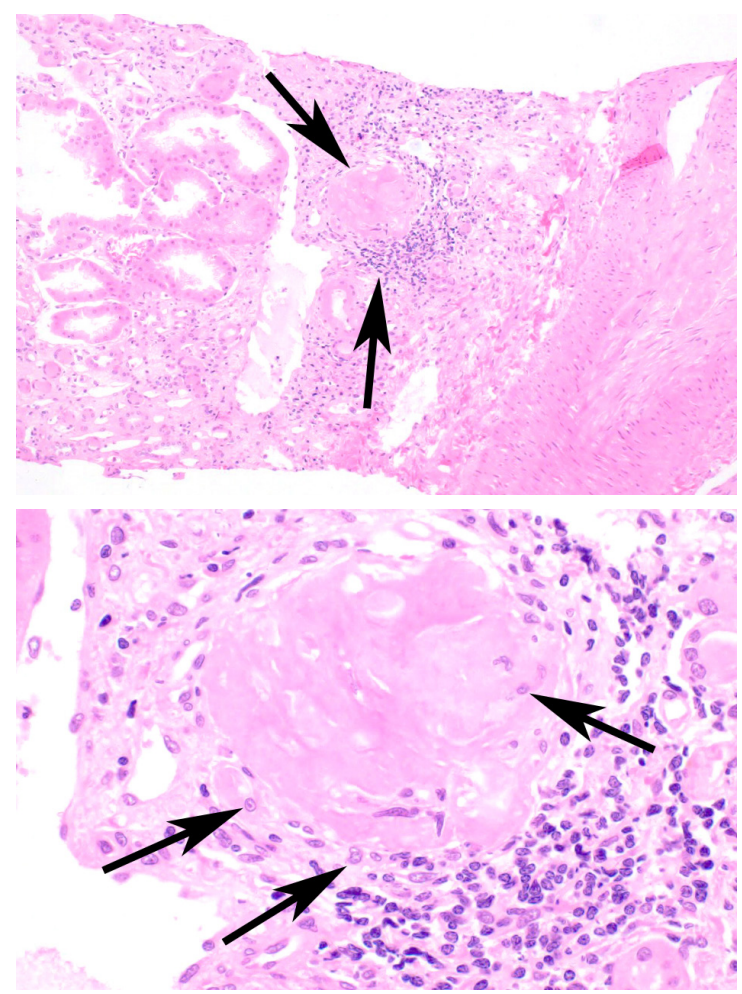

Figure 2: Histologic findings (A) Vague granuloma (arrow) (H-E stain, $\times 200$ ); (B) Granuloma showed a central necrosis and epithelioid cells (arrows) with some lymphocytes few neutrophils $(\mathrm{H}-\mathrm{E}$ stain, $\times 400)$ 


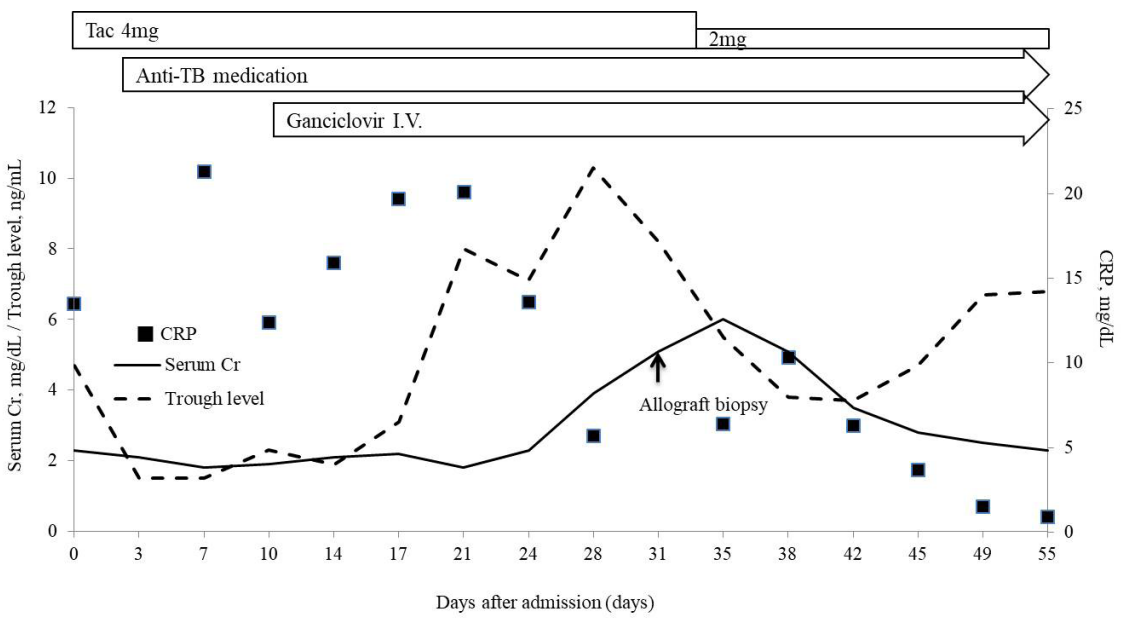

Cr: Creatinine; CRP: C-reactive protein; Tac: Tacrolimus; TB: Tuberculosis

Figure 3: Timeline of patient's management and hospital course
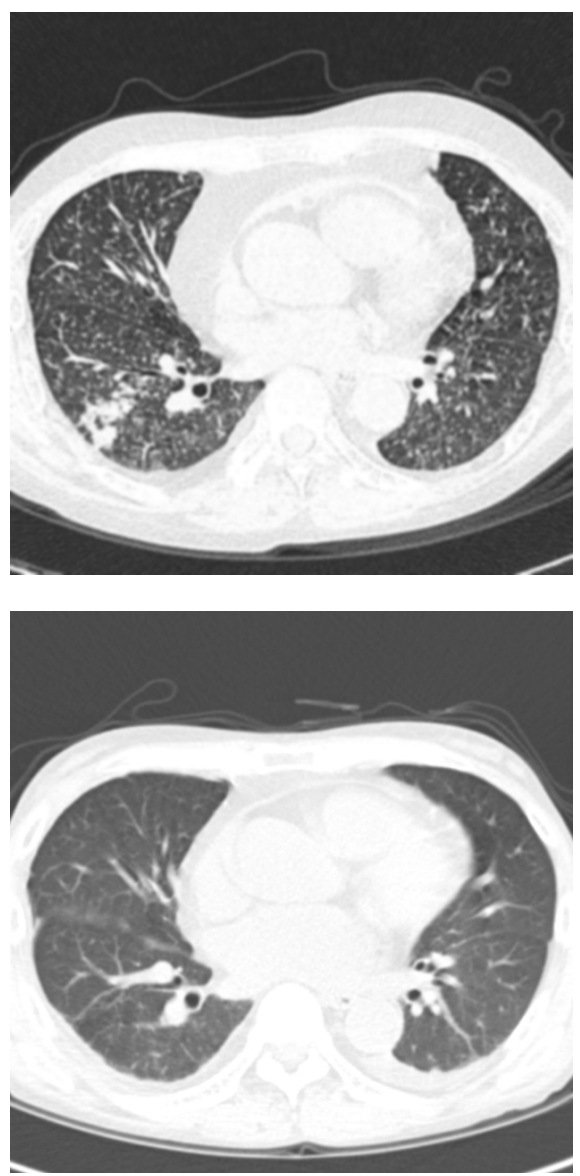

Figure 4: Chest CT findings (A) Pre-treatment: Multiple tiny nodules in both lungs; (B) 1-year after TB-treatment: Complete resolution of multiple nodules

\section{Discussion}

Tuberculosis (TB) is not uncommon infection in organ transplant recipients. Recently, with increased the incidence and prevalence of active or latent infection in community, incidence of TB in transplant recipient can be affected [3,4]. Contrary to typical pulmonary TB in general population, TB in transplant recipients frequently presented unusual feature, with mortality rates of 10$40 \%[1,3]$. Thus, early diagnosis and treatment is mandatory for improving graft and patient outcome in these patients.

For active TB infection, the prevalence among solid organ transplant patients in most developed countries is $1.2-6.4 \%$, while the prevalence in transplant patients in highly endemic areas has been reproted to be up to $15 \%[1,4,5]$. Approximately $30-50 \%$ of TB after transplantation is extra-pulmonary or disseminated. Pulmonary involvement in transplant recipients is often diffuse pulmonary infiltrations rather than cavitary lesion which commonly seen in immunocompetent patients [3]. In Spanish retrospective matched cohort study, disseminated tuberculosis was more frequent in transplant patients compared to non transplant patients 
(23\% vs. $5 \%)$ and because of atypical presentation, time from clinical suspicion of TB to definitive diagnosis longer in transplant patients than in non transplant patients [3]. In Turkish retrospective study, 2.8\% of transplant patients diagnosed of TB and 55.5\% had extrapulmonary TB [6]. In our case, the patient complained of dry coughing for 2 weeks and chest radiography showed miliary pattern. Initial clinical presentation was not implicated allograft involvement. There was no pain on allograft and kidney function was not changed significantly with negative for AFB and WBC in urinary samples. Combination of clinical, laboratory and radiologic findings, the initial diagnosis was miliary TB. Because of complicated clinical course with CMV viremia, it was not easy to diagnosis of allograft involvement. Walker JF et al. [7] first decribed a case of disseminated TB and allograft involvement, successfully treated without graft loss. George $\mathrm{P}$ et al. reported a case of allograft TB with unusual presentation [8]. The patient complaint of nonspecific gastrointestinal symptom with mild fever and laboratory findings showed allograft dysfunction without evidence of TB infection in urinary tract. Khaira A et al. [9] reported three cases of kidney allograft TB and one of three lost graft function. In their report, all of patients presented with fever of unknown origin with or without allograft dysfunction [9].

Several clinical studies suggested risk factors for TB development in transplant patients. Suggested risk factors are certain immunosuppressive drugs, diabetes mellitus, and coexisting infections such as CMV infection, deep mycosis, Pneumocystis jiroveci pneumonia and Norcadia infection [5]. In this case, because the patient had a history of pulmonary TB after first KT, he received 6-month INH prophylaxis. He diagnosed of chronic rejection 4months after KT and the intensity of immunosuppression was not adjusted. As a result the patient received triple immunosuppressive treatment for almost 9 years. This could be a risk factor for late CMV pneumonia and followed by disseminated TB infection despite of discontinuation of MMF after CMV infection. Recently Cobelens et al. [10] suggested that immunologically active CMV infection can act as second-hit or precipitating factor for progression of latent TB infection to TB disease. Thus, there is a possibility that prior CMV infection may trigger TB reactivation in our patient. In Turkish study, two patient diagnosed of TB at the time of 100 and 150 months after KT, respectively [6].

Treatment of TB usually consisted of 4-drug regimen with 6-months duration. Extrapulmonary TB, such as bone marrow, or severe disseminated disease are treated for a total of six to nine months and central nerve system disease warrants treatment duration of nine to twelve months [11]. In our case, the patient was treated with 12-months duration for disseminated disease involving allograft considering previous history of pulmonary TB and CMV co-infection. In the transplant population, TB contributes to graft dysfunction, both through direct effects on th graft and as a result of drug interactions, and thereby increases mortality $[3,5,11]$. In Spanish study TB related mortality was higher among transplant patients than among non transplant patients [3]. Ersan et al. reported that one out of 12 patients died of dissemination of TB and one patient returned to HD [6]. In the report of Khaira A et al., one of third patients lost graft function [9]. In this case, TB was all sensitive for 4 drugs and the patient was treated without serious drug side effects.

\section{Conclusion}

In summary, we report the case of disseminated TB, involving lung and allograft, 10 years after kidney transplantation. Because of complicated clinical course, diagnosis of allograft infection was delayed however, successfully treated with 4 -drugs regimen. With increasing prevalence of latent TB in community, efforts should be made to prevent TB infection by recognition and treatment of latent TB in transplant candidates.

\section{Acknowledgement}

This research was supported by Bisa Research Grant of Keimyung University in 2013.

\section{Conflicts of interest}

No potential conflicts of interest relevant to this article were reported.

\section{References}

1. Subramanian A, Sorman S (2009) Mycobacterium tuberculosis in solid organ transplant recipients. Am J Transplant 9: S57-62.

2. Aguado JM, Herraro JA, Gavalda J, Torre-Cisneros J, Blanes M, et al. (1997) Clinical presentation and outcome of tuberculosis in kidney, liver, and heart transplant recipients in Spain. Spanish Transplantation Infection Study Group, GESITRA. Transplantation 63: 1278-86.

3. Benito N, Garcia-Vazquez E, Horcajada JP, Gonzalez J, Oppenheimer F, et al. (2015) Clinical features and outcomes of tuberculosis in transplant recipients as compared with the general population: a retrospective matched cohort study. Clin Microbiol Infect 21: 651-8.

4. Kim HJ (2006) Current situation of tuberculosis and its cost in Korea. J Korean Med Assoc 49: 762-72.

5. Munoz P, Rodriguez C, Bouza E (2005) Mycobacterium tuberculosis infection in recipients of solid organ transplants. Clin Infect Dis 40: 581-7.

6. Ersan S, Celik A, Atila K, Sifil AA, Cavdar C, et al. (2011) Tuberculosis in renal transplant recipients. Ren Fail 33: 753-7.

7. Walker JF, Cronin CJ, O’Neill S, McNulty J, Hanson JS, et al. (1982) Tuberculosis affecting a cadaveric renal allograft. Clin Nephrol 17: 262-5.

8. George P, Pawar B, Calton N (2008) Tuberculosis in a renal allograft: A successful outcome. Saudi J Kidney Dis Transplant 19: 790-2.

9. Khaira A, Bagchi S, Sharma A, Mukund A, Mahajan S, et al. (2009) Renal allograft tuberculosis: report of three cases and review of literature. Clin Exp Nephrol 13: 392-6.

10. Cobelens F, Nagelkerke N, Fletcher H (2018) The convergent epidemiology of tuberculosis and human cytomegalovirus infection. Version 2. F1000Res 7: 280. 11. Subramanian AK, Morris MI (2013) Mycobacterium tuberculosis infections in solid organ transplantation. Am J Transplant 13: 68-76. 


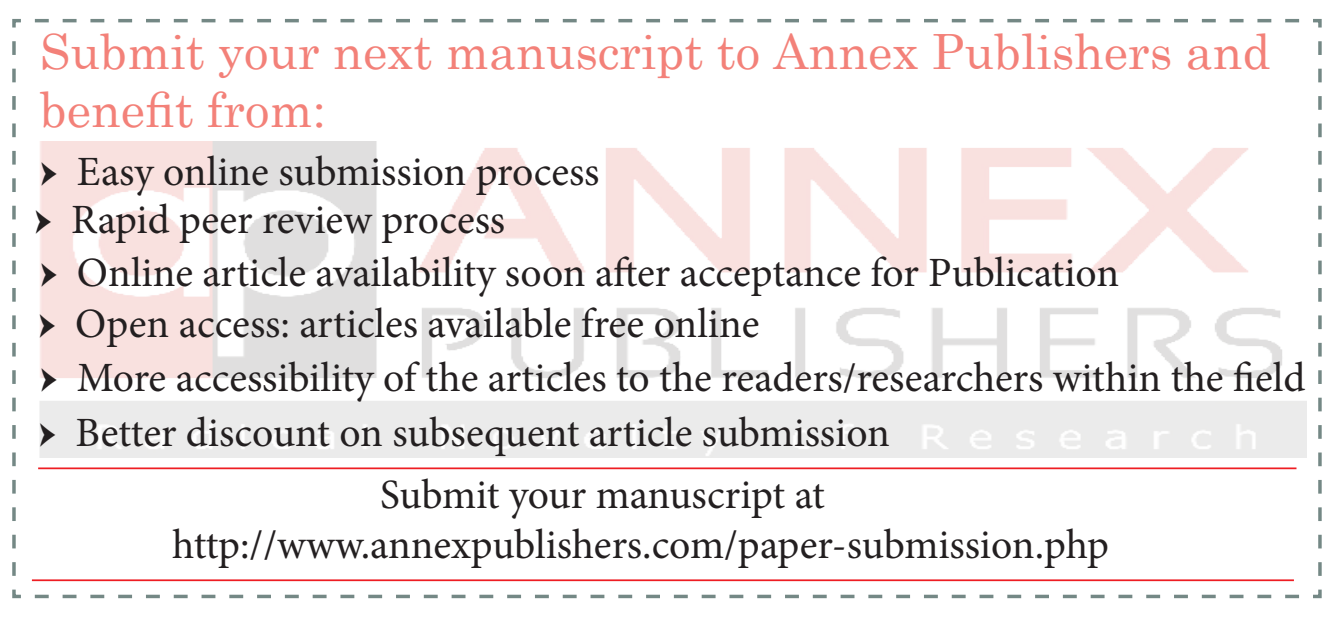

